\title{
Periodic mechanical stress induces the extracellular matrix expression and migration of rat nucleus pulposus cells by upregulating the expression of intergrin $\alpha 1$ and phosphorylation of downstream phospholipase $\mathbf{C} \gamma \mathbf{1}$
}

\author{
GONGMING GAO ${ }^{1 *}$, JIN HE ${ }^{2 *}$, LUMING NONG ${ }^{1}$, HUA XIE $^{2}$, \\ YONGJING HUANG $^{1}$, NANWEI XU ${ }^{1}$ and DONG ZHOU ${ }^{1}$ \\ ${ }^{1}$ Department of Orthopedics, Changzhou Second Hospital Affiliated to Nanjing Medical University, Changzhou, \\ Jiangsu 213003; ${ }^{2}$ Department of Orthopedics, Jintan People's Hospital Affiliated to Jiangsu University, \\ Jintan, Jiangsu 213200, P.R. China
}

Received August 6, 2015; Accepted July 8, 2016

DOI: $10.3892 / \mathrm{mmr} .2016 .5549$

\begin{abstract}
Intervertebral disk degeneration (IDD) is a major cause of low back pain and an important socioeconomic burden. Degradation of the extracellular matrix (ECM) of nucleus pulposus (NP) cells in the interverterbal disk is important for IDD. Stress of a suitable frequency and amplitude promotes the synthesis of the ECM of NP cells, however, the associated mechanisms remain to be fully elucidated The present study aimed to investigate the effect of integrin $\alpha 1$ on the migration and ECM synthesis of NP cells under soft periodic mechanical stress. Rat NP cells were isolated and plated onto slides, and were then treated with or without the use of a periodic mechanical stress system. The expression levels of integrin $\alpha 1$, $\alpha 5$ and $\alpha \mathrm{v}, \mathrm{ECM}$ collagen 2A1 (Col2A1) and aggrecan, and the phosphorylation of phospholipase C- $\gamma 1$ (PLC $\gamma 1)$ were measured using reverse transcription-quantitative polymerase
\end{abstract}

Correspondence to: Dr Luming Nong, Department of Orthopedics, Changzhou Second Hospital Affiliated to Nanjing Medical University, 29 Xinglong Alley, Changzhou, Jiangsu 213003, P.R. China

E-mail: lnong88125381@sina.com

Mr. Hua Xie, Department of Orthopedics, Jintan People's Hospital Affiliated to Jiangsu University, 16 Nanmen Street, Jintan, Jiangsu 213200, P.R. China

E-mail: lnong88125381@sina.com

*Contributed equally

Abbreviations: IDD, intervertebral disk degeneration; ECM, extracellular matrix; NP, nucleus pulposus; Col2A1, collagen 2A1; PLC $\gamma 1$, phospholipase C- $\gamma 1$; IVD, intervertebral disc

Key words: nucleus pulposus cells, periodic mechanical stress, extracellular matrix, integrin $\alpha 1$, phospholipase C- $\gamma 1$, phosphorylation, migration chain reaction and western blot analyses. Cell migration was assayed using a scratch experiment. The results showed that exposure to periodic mechanical stress significantly induced the mRNA expression levels of Col2A1 and aggrecan, cell migration, mRNA expression of integrin $\alpha 1$ and phosphorylation of PLC- $\gamma 1$ of the NP, compared with the control $(\mathrm{P}<0.05)$. Inhibition of the PLC $\gamma 1$ protein by $\mathrm{U} 73122$ significantly decreased the ECM expression under periodic mechanical stress $(\mathrm{P}<0.05)$. Small interfering RNA-mediated integrin $\alpha 1$ gene knockdown suppressed the mRNA expression levels of Col2A1 and aggrecan, and suppressed the migration and phosphorylation of PLC $\gamma 1$ of the NP cells under periodic mechanical stress, compared with the control $(\mathrm{P}<0.05)$. In conclusion, periodic mechanical stress induced ECM expression and the migration of NP cells via upregulating the expression of integrin $\alpha 1$ and the phosphorylation of downstream PLC $\gamma 1$. These findings provide novel information to aid the understanding of the pathogenesis and development of IDD.

\section{Introduction}

Intervertebral disc (IVD) degeneration (IDD) is the primary cause of low back pain, which is becoming an important socioeconomic burden. The IVD is composed of the nucleus pulposus (NP), annulus fibrosus and cartilage endplate, and contain extracellular matrix (ECM), which includes collagens (predominantly type-II collagen in NP) and proteoglycans (predominantly aggrecan) (1). Through the stimulation of various mechanical and biochemical signals, these ECM components may regulate cell morphology, phenotype, differentiation and ECM production of NP cells (2). The degradation of ECM in IVDs, particularly in the NP, is an important cause of IDD (1).

In the body, NP cells are in a complex mechanical environment, and their functions are affected by mechanical factors (3-5). Under physiological conditions, the stress in the human intervertebral space varies with postures between 0.1-1.1 MPa (6). Mechanical stress is important in the 
homeostasis of ECM in IVD cells. Periodic mechanical stress with low frequency and amplitude promotes the synthesis of ECM of NP cells and inhibits its degradation (7); whereas severe stress directly induces the dysfunction of energy metabolism and apoptosis of NP cells (8), possibly causing spinal diseases, including IDD. However, the mechanisms underlying the effects of mechanical stress on the behaviors of NP cells remains to be fully elucidated.

Integrins are a family of adhesion proteins on the cell surface, which are important for cell adhesion, proliferation, apoptosis and migration $(9,10)$. Integrins transfer extracellular mechanical signals into intracellular chemical signals, regulating cellular metabolism via the downstream signaling pathways (11). Structurally, integrins are heterodimers containing $\alpha$ and $\beta$ units, which jointly interact with various ligands. There are 18 types of $\alpha$ subunits and eight types of $\beta$ subunits, constituting 24 types of integrins (12). Previous studies have shown the presence of various integrin subunits in NP regions, including $\alpha 1, \alpha 2, \alpha 3, \alpha 5, \alpha 6, \alpha \mathrm{V}, \beta 1$ and $\beta 4$ subunits (13-17). However, until now, which and how these integrins mediate the regulatory role of periodic mechanical stress in the synthesis and migration of ECM in NPs remain to be fully elucidated.

The phosphorylation of phospholipase C- $\gamma 1$ (PLC $\gamma 1)$ protein, a serine theronine kinase belonging to the phospholipase $\mathrm{C}$ family, is ubiquitous in various cells to regulate processes, including cell adhesion, migration and ECM synthesis (18-20). In our previous study, it was demonstrated that, in chondrocytes, periodic mechanical stress activated PLC $\gamma 1$ by Src through phosphorylation at the site of $\mathrm{Tyr}^{783}$ $\left(\mathrm{PLC} \gamma 1-\mathrm{Tyr}^{783}\right.$ ) to promote chondrocyte area expansion and migration, partially via the mitogen-activated protein kinase kinase 1/2-extracellular signal-regulated kinase 1/2 pathway $(21,22)$. It was also reported that periodic mechanical stress induced the expression of ECM collagen II (Col-2) and proteoglycan, and induced the phosphorylation of PLC $\gamma 1$ protein in NPs, whereas treatment with U73122, an inhibitor of PLC $\gamma 1$, significantly suppressed the cyclic stress-induced expression of ECM (23). These results indicated that PLC- $\gamma 1$ may mediate the regulatory role of periodic mechanical stress in the expression of ECM in NPs. However, how PLC $\gamma 1$ is involved in this process remains to be fully elucidated.

The current study aimed to investigate whether integrins and PLC $\gamma 1$ have regulatory roles in periodic mechanical stress in NP cells. The present study indicated that the periodic mechanical stress-induced expression of ECM and migration of NP cells was mediated by the expression of integrin $\alpha 1$ and phosphorylation of downstream PLC $\gamma 1$. These findings provide novel clues for investigating the mechanisms underlying the effects of periodic mechanical stress on regulation of the behaviors of NP cells, and to understand the pathogenesis and development of IDD.

\section{Materials and methods}

Isolation and culturing of NP cells. NP cells were isolated and cultured, as described previously (24). A total of 60 male Sprague-Dawley rats (4-week-old) were obtained from the Animal Center of Nanjing Medical University (Nanjing, China), they were maintained in standard conditions of $24 \pm 1^{\circ} \mathrm{C}$, with a relative humidity of $50 \%$. They had access to food and water ad libitum and were kept under a 12-h light/dark cycle. The rats were sacrificed by cervical dislocation, following which the thoracic and lumbar spines were collected under sterile conditions. Following removal of the surrounded ligament and soft tissues, the IVDs were rapidly cut open from the ventral side and digested in $1.5 \%$ type II collagenase (Gibco; Thermo Fisher Scientific, Inc., Waltham, MA, USA) at $37^{\circ} \mathrm{C}$ for $2 \mathrm{~h}$, followed by filtration through a 200 mesh strainer. The resultant cells were cultured in Dulbecco's modified Eagle's medium-F12 medium (Gibco; Thermo Fisher Scientific, Inc.) supplemented with $10 \%$ fetal bovine serum (FBS; GE Healthcare Life Sciences Hyclone Laboratories, Logan, UT, USA) in a BB5060 incubator (Heraeus, Hanau, German) at $37^{\circ} \mathrm{C}$ and $5 \% \mathrm{CO}_{2}$. The cells were subcultured at a confluence of $80 \%$, and cells in the second passage were used for the following experiments. The surgery on the animals was conducted by Hangzhou Hibio Technology Co., Ltd. (Hangzhou, China) and approved by their Institutional Animal Care and Use Committee.

Cell treatment. A periodic mechanical stress system was used, as previously described (21). The periodic mechanical stress culturing system (Taixing Experimental Instrument Factory, Jiangsu, China), comprised a reciprocating boost pump and a culture chamber, which provided a periodic mechanical stress with a pressure of $0-0.3 \mathrm{MPa}$ and frequency of $0-1 \mathrm{~Hz}$. The cells $\left(1 \times 10^{5}\right.$ cells $\left./ \mathrm{ml}\right)$ were plated on slides $(25 \times 25 \mathrm{~mm})$, and then underwent periodic mechanical stress treatment of $0-0.2 \mathrm{MPa}$ and $0.1 \mathrm{~Hz}$ for $6 \mathrm{~h}$ (stress group) or were not exposed to stress (control group). The cells were then collected for detection of the expression levels of integrin $\alpha 1, \alpha 5, \alpha \mathrm{V}$, collagen 2A1 (Col2A1) and aggrecan, the phosphorylation of PLC $\gamma 1$ at $\operatorname{Tyr}^{783}\left(\mathrm{PLC} \gamma 1-\mathrm{Tyr}^{783}\right)$ and cell migration of the NPs.

In certain experiments, NPs were transfected with either integrin $\alpha 1$ small interfering (si)RNA (siRNA group) or negative control siRNA (NC group), or remained untransfected (control group) prior to the administration of periodic mechanical stress (0-0.2 MPa; 0.1 Hz; $6 \mathrm{~h}$ ). NPs were also pretreated with U73122 (Gibco; Thermo Fisher Scientific, Inc.), an inhibitor of PLC $\gamma 1$, in DMSO at a concentration of $10 \mu \mathrm{M}$ (U73122 group) or with DMSO alone (control group) prior to the administration of periodic mechanical stress. After $6 \mathrm{~h}$ of stress, the cells were collected for various assays.

Cell transfection. The siRNA for integrin $\alpha 1$ was as follows: Sense 5'-GGUCGGGAUUGUACAGUAUGGTT-3' and antisense 5'-CCAUACUGUACAAUCCCGACCTT-3'; The NC siRNA was as follows: Sense 5'-UUCUCCGAACGUGUC ACGUTT-3' and antisense 5'-ACGUGACACGUUCGGAGA ATT-3'. The siRNA and the negative control were synthesized by Shanghai GenePharma Co., Ltd. (Shanghai, China).

For transfection, $75 \mathrm{pM}$ of siRNA or NC, and $7.5 \mu \mathrm{l}$ lipofectamine 2000 reagent (Invitrogen; Thermo Fisher Scientific, Inc.) were resolved in $50 \mu \mathrm{l}$ opti-MEM medium (Gibco; Thermo Fisher Scientific, Inc.), respectively, mixed for $5 \mathrm{~min}$ and added to the cells on slides $(100 \mu \mathrm{l}$ for each slide; $1 \times 10^{5}$ cells $/ \mathrm{ml}$ ). After 6 hours at $37^{\circ} \mathrm{C}$, the medium was replaced. The cells were collected for western blot analysis to 
confirm successful transfection, and then underwent periodic mechanical stress treatment.

Western blot analysis. The cells were collected and washed with phosphate-buffered saline (PBS), and added to RIPA lysis buffer (Beyotime Institute of Biotechnology, Nantong, China) on ice for $5 \mathrm{~min}$. The cell lysate was centrifuged at $14,000 \mathrm{~g}$ for $5 \mathrm{~min}$ at $4^{\circ} \mathrm{C}$, and the supernatant was collected. The concentration of the resultant total protein was determined using a bicinchoninic acid assay (Beyotime Institute of Biotechnology), and the protein was denatured and samples (50 $\mu \mathrm{g}$ ) were separated by sodium dodecyl sulfate polyacrylamide gel electrophoresis with an equal quantity of total protein for each sample. The proteins in the gel were transferred onto polyvinylidene fluoride membranes, blocked and incubated with primary antibodies as follows: Goat polyclonal anti-integrin $\alpha 1$ (1:1,000; cat. no. sc-6584, Santa Cruz Biotechnology, Inc., Dallas, TX, USA), mouse polyclonal anti-PLC $\gamma 1$ (1:1,000; cat. no. ab16955; Abcam, Cambridge, MA, USA), rabbit polyclonal anti-PLC $\gamma 1-\operatorname{Tyr}^{783}(1: 1,000$; cat. no. 2821 ; Cell Signaling Technology, Inc., Danvers, MA, USA) and rabbit polyclonal anti-GAPDH (1:5,000; cat. no. AP0063; Bioworld Technology, Inc., Louis park, MN, USA) at $4^{\circ} \mathrm{C}$ overnight. Following washing of the membrane PBS with Tween 20, the goat anti-rabbit IgG-horseradish peroxidase (HRP; cat. no. BS13278; Bioworld Technology, Inc.), goat anti-mouse IgG-HRP (cat. no. BS12478; Bioworld Technology, Inc.) or rabbit anti-goat IgG-HRP (cat. no. sc-2768; Santa Cruz Biotechnology, Inc.) secondary antibodies were then added at room temperature for $1 \mathrm{~h}$. Following washing, the membrane was visualized using Immobilon ${ }^{\mathrm{TM}}$ Western Chemiluminescent HRP substrate reagent (EMD Millipore, Billerica, MA, USA). The blots were scanned using a Bio-Rad Gel Doc Imaging System (Bio-Rad Laboratories, Inc., Hercules, CA, USA), and the band densities were quantified and compared using QuantityOne software (Bio-Rad Laboratories, Inc.). Expression of the target protein was calculated as the band density of the target protein normalized to that of GAPDH.

Reverse transcription-quantitative polymerase chain reaction (RT-qPCR) analysis. The total RNA was extracted from the cells using TRIzol regent (Invitrogen; Thermo Fisher Scientific, Inc.), and transcribed into cDNA using a PrimeScript RT Master Mix kit (Takara Bio, Inc., Shiga, Japan). The primers for integrin $\alpha 1$, integrin $\alpha 5$, integrin $\alpha V$, aggrecan and Col2al were as follows: sense 5'-GGGCTACTGCTGCTAATGCT-3' and antisense 5'-GGCCTTTTGAAGAATCCAATC-3' for integrin $\alpha 1$; sense 5'-AGCTGCATTTCCGAGTCTG-3' and antisense 5'-CTCACACTGAAGGCTGAACG-3' for integrin $\alpha 5$; sense 5'-GGTGTGGATCGAGCTGTCTT-3' and antisense CAAGGCCAGCATTTACAGTG-3' for integrin $\alpha V$; sense 5'-CCCTACCCTTGCTTCTCCA-3' and antisense 5'-CTTGAGAGGCACTCATCAATGT-3' for agrecan; sense 5'-GACCCCCAGGTTCTAATGG-3' and antisense 5'-GCA CCTTTGGGACCATCTT-3' for Col2A1; and sense 5'-GCA GAAGGAGATTACTGCCCT-3' and antisense 5'-GCTGAT CCACATCTGCTGGAA-3' for $\beta$-actin. The primers were synthesized by Shanghai GenePharma Co., Ltd. RT-qPCR analysis was performed using a SYBR Premix Ex Taq II kit (Takara Bio, Inc.) and a StepOnePlus Real-Time PCR system
(Thermo Fisher Scientific, Inc.). The reaction system $(20 \mu \mathrm{l})$ contained $10 \mu \mathrm{l} 2 \mathrm{X}$ SYBR Premix Ex Taq II, $0.4 \mu \mathrm{l}$ forward primer $(10 \mu \mathrm{M}), 0.4 \mu \mathrm{l}$ reverse primer $(10 \mu \mathrm{M}), 0.4 \mu \mathrm{l}$ 50X ROX Reference Dye, $2 \mu \mathrm{l}$ DNA sample, and $6.8 \mu \mathrm{l}$ distilled water. Thermocycling conditions were as follows: $95^{\circ} \mathrm{C}$ for $30 \mathrm{sec}$; 40 cycles of $95^{\circ} \mathrm{C}$ for $5 \mathrm{sec}$ and $60^{\circ} \mathrm{C}$ for $30 \mathrm{sec}$. The results were quantified using the $2^{-\Delta \Delta \mathrm{Cq}}$ method (19).

Cell migration assay. A scratch test is an effective means for the assessment of the migration capacities of NP cells (25). Following treatment, scratches were introduced vertically on the bottom of the slides, with the fine end of $200 \mu \mathrm{l}$ tips. The medium was then removed, and the slides were washed with PBS three times to remove the detached cells. Serum-free medium was then added for culturing in an incubator at $37^{\circ} \mathrm{C}$, $5 \% \mathrm{CO}_{2}$ for $4 \mathrm{~h}$. The slides were observed under a CKX31 microscope (Olympus, Tokyo, Japan), and images were captured under three optimal fields at 0 and $4 \mathrm{~h}$ following introduction of the scratch with the tips. Cell migration was analyzed using ImageJ 1.43 software (imagej.nih.gov). The cell migration distance $(\mu \mathrm{m})$ was calculated as the scratch width (shown as the distance between the two dotted lines in Fig. 1) at $0 \mathrm{~h}$ minus the scratch width $4 \mathrm{~h}$ following the introduction of scratch injury.

Statistical analysis. Data are expressed as the mean \pm standard deviation and analyzed using SPSS 13.0 software (SPSS Inc., Chicago, IL, USA). The data between groups were analyzed using an unpaired t-test. $\mathrm{P}<0.05$ was considered to indicate a statistically significant difference.

\section{Results}

Periodic mechanical stress significantly induces the $m R N A$ expression of ECM Col-2Al and aggrecan, and promotes the migration of NP cells. Compared with the control, which was not exposed to stress treatment, periodic mechanical stress (0.2 MPa; $0.1 \mathrm{~Hz} ; 6 \mathrm{~h})$ significantly induced the mRNA expression of ECM Col2A1 and aggrecan, as determined using RT-qPCR analysis $(\mathrm{P}<0.05$, Fig. 1A), and promoted the migration of NP cells, determined in scratch experiments $(\mathrm{P}<0.05$, Fig. 1B)

Periodic mechanical stress significantly upregulates the $m R N A$ expression of integrin $\alpha 1$ and induces the phosphorylation of PLC $\mathrm{C} 1$ in NP cells. The RT-qPCR analysis showed that periodic mechanical stress significantly induced the mRNA expression of integrin $\alpha 1$, but inhibited the expression levels of intergrin $\alpha 5$ and $\alpha \mathrm{V}$, compared with the control $(\mathrm{P}<0.05$; Fig. 2A). Periodic mechanical stress also significantly induced the phosphorylation of PLC- $\gamma 1$ ( $\mathrm{P}<0.05$; Fig. 2B).

Phosphorylation of PLC $\gamma 1$ is required for the periodic mechanical stress-induced expression of ECM. To examine whether the phosphorylation of PLC $\gamma 1$ is involved in the periodic mechanical stress-induced expression of ECM, the NP cells were pretreated with U73122 prior to the administration of periodic mechanical stress. Pretreatment with U73122 significantly inhibited the phosphorylation of PLC $\gamma 1$ in the NP cells $(\mathrm{P}<0.05$; Fig. 3A), and suppressed the mRNA expression 
A

Aggrecan

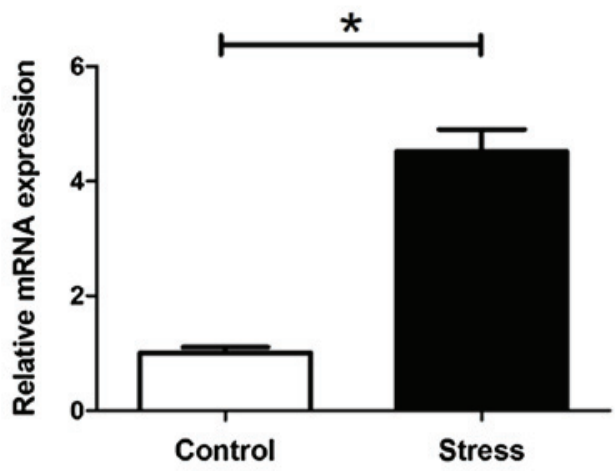

B

0h

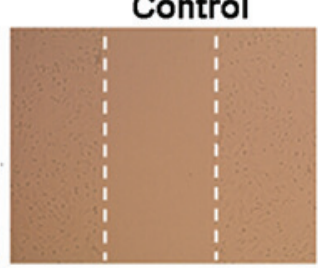

$4 h$

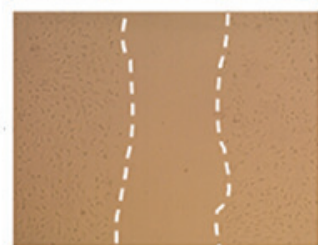

Stress
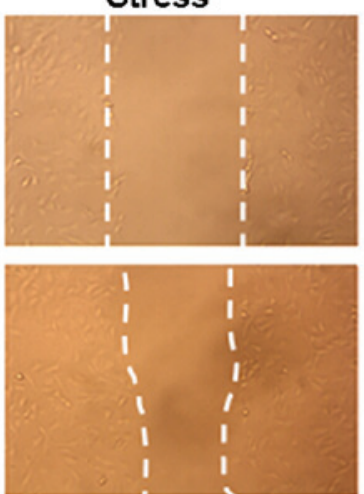

Col2AI
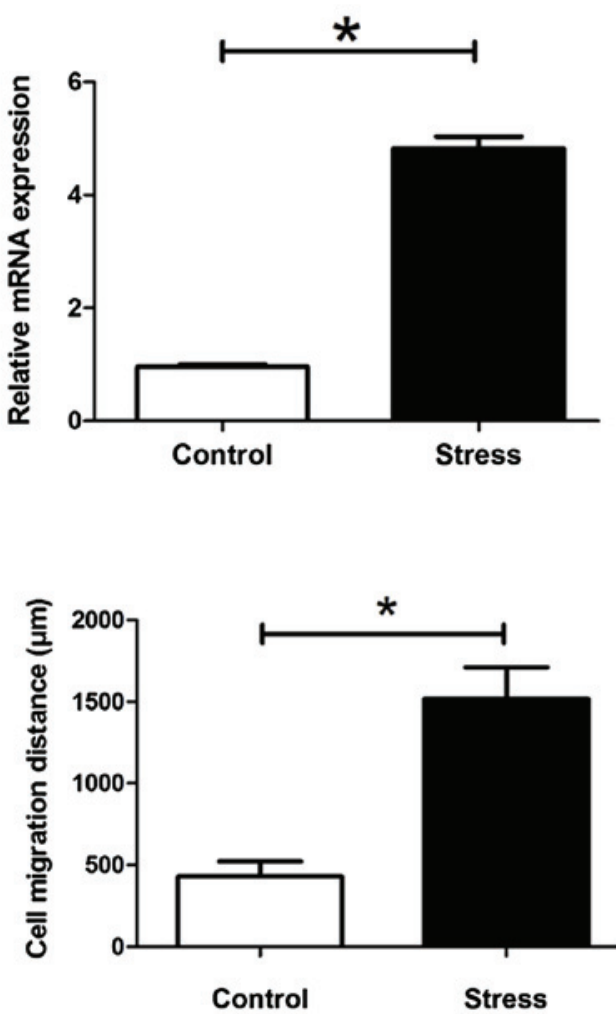

Figure 1. Periodic mechanical stress significantly induces the mRNA expression of Col2A1 and aggrecan, and promotes nucleus pulposus cell migration. Cells were exposed to periodic mechanical stress (stress) or remained untreated (control). (A) mRNA expression levels of Col2A1 and aggrecan were analyzed by reverse transcription-quantitative polymerase chain reaction analysis. (B) Migration was determined by scratch experiments. Dotted lines indicate scratch widths (magnification, $\mathrm{x} 200$ ). Data are expressed as the mean \pm standard deviation. ${ }^{*} \mathrm{P}<0.05$, compared with the control ( $\mathrm{n}=3$ ). Col2A1, collagen $2 \mathrm{~A} 1$.

A

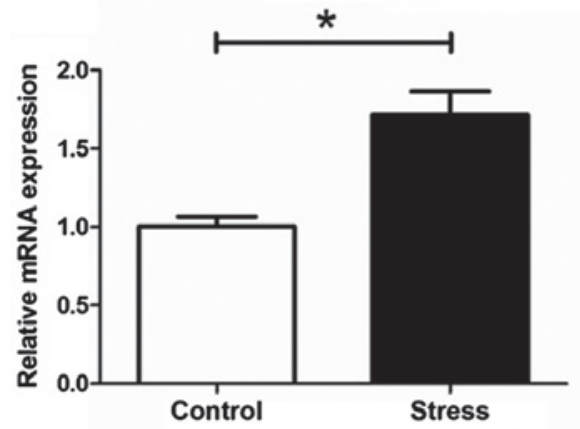

B
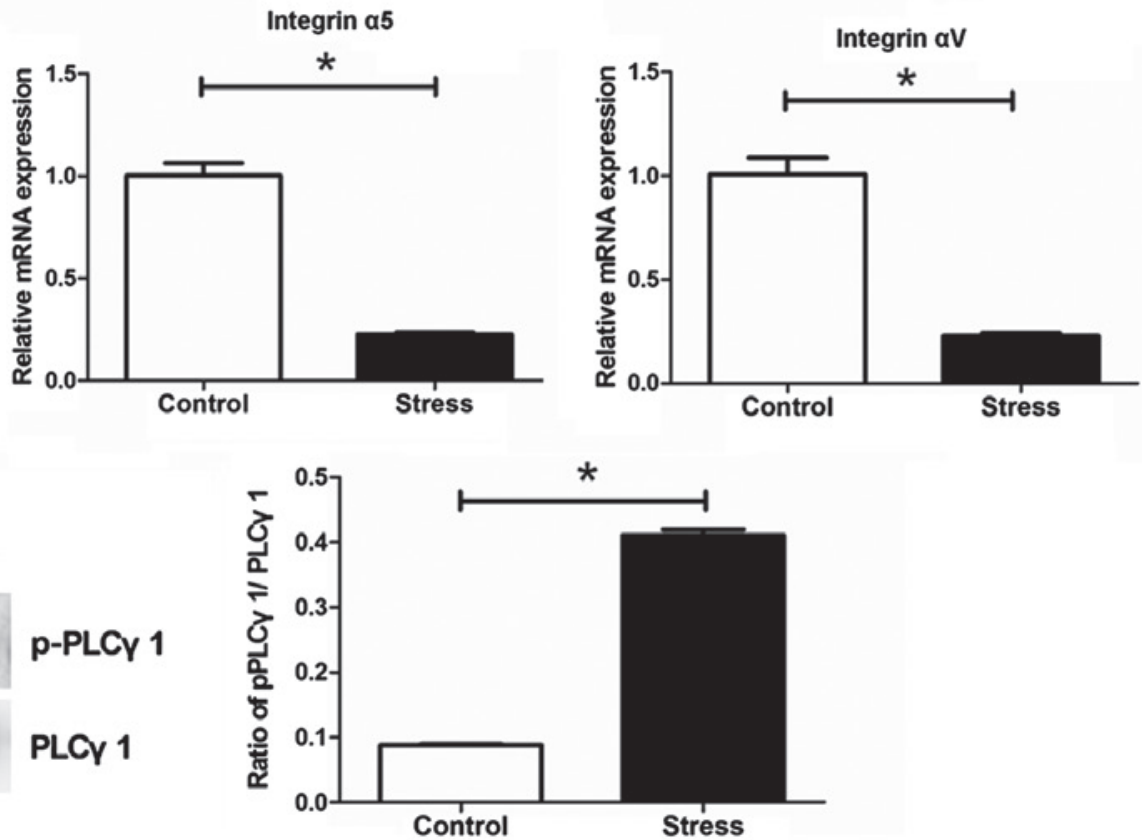

Figure 2. Periodic mechanical stress significantly induces the mRNA expression of integrin $\alpha 1$ and phosphorylation of PLC $\gamma 1$ in nucleus pulposus cells. Cells exposed to periodic mechanical stress (stress) or not (control) were analyzed for the (A) mRNA levels of integrin $\alpha 1, \alpha 5$ and $\alpha \mathrm{V}$ by reverse transcription-quantitative polymerase chain reaction analysis. (B) Phosphorylation of PLC $\gamma 1$ was analyzed by western blot analysis. Data are expressed as the mean \pm standard deviation. "P<0.05, compared with the control (n=3). PLC $\gamma 1$, phospholipase C- $\gamma 1$; p-PLC $\gamma 1$, phosphorylated PLC $\gamma 1$. 
A

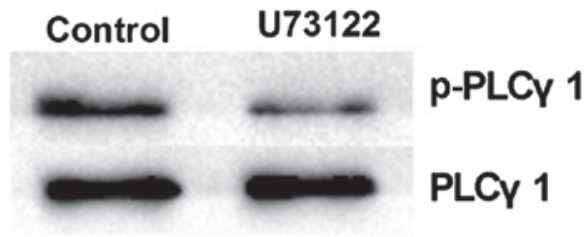

B

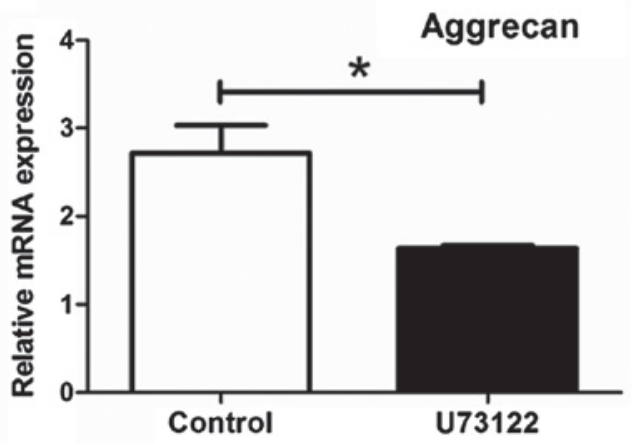

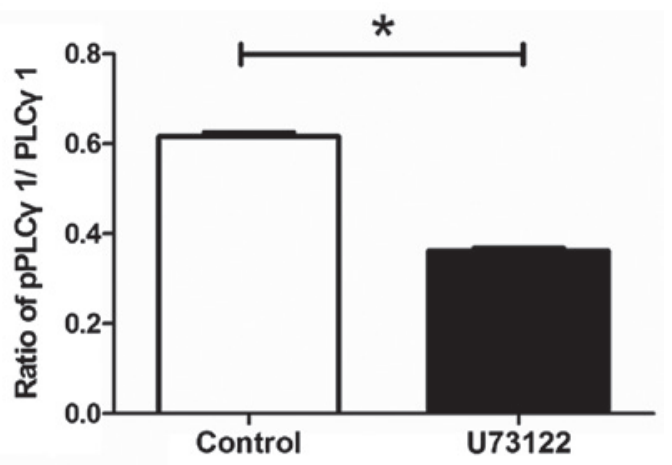

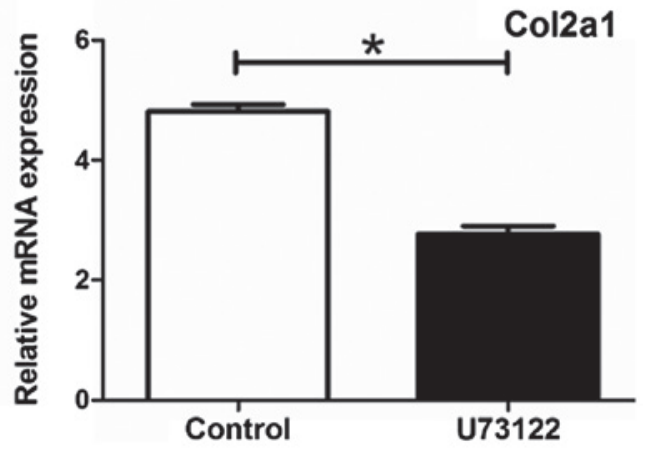

Figure 3. Phosphorylation of PLC $\gamma 1$ is required for periodic mechanical stress-induced extracellular matrix expression. Nucleus pulposus cells were pretreated with U73122 or DMSO (control) prior to the administration of periodic mechanical stress. The (A) ratio of p-PLC $\gamma 1$ to PLC $\gamma 1$, and (B) mRNA expression levels of Col2A1 and aggrecan were analyzed. Data are expressed as the mean \pm standard deviation. ${ }^{*} \mathrm{P}<0.05$, compared with the control ( $=3$ ). PLC $\gamma 1$, phospholipase C- $\gamma 1$; p-PLC $\gamma 1$, phosphorylated PLC $\gamma 1$; Col2A1, collagen 2A1.

levels of Col2A1 and aggrecan in the NP cells under periodic mechanical stress $(\mathrm{P}<0.05$; Fig. 3B).

siRNA-based inhibition of integrin $\alpha 1$ suppresses the $m R N A$ expression of Col2A1 and aggrecan, the migration of NPs and the phosphorylation of PLC $\gamma 1$ under periodic mechanical stress. As the results of the present study showed that periodic mechanical stress induced the mRNA expression of integrin $\alpha 1$ in NP cells under periodic mechanical stress, to determine whether integrin $\alpha 1$ is involved in the regulatory effect of periodic mechanical stress on NP cells, integrin $\alpha 1$ siRNA was transfected into the NP cells. As shown in Fig. 4A, transfection with integrin $\alpha 1$ siRNA resulted in a significant decrease in the protein expression of integrin $\alpha 1$, as detected using western blot analysis $(\mathrm{P}<0.05)$, confirming the inhibition of integrin $\alpha 1$ in the NP cells of the siRNA group. Compared with the untransfected NP cells or the cells transfected with NC siRNA, transfection with integrin $\alpha 1$ siRNA suppressed the mRNA expression levels of Col2A1 and aggrecan (Fig. 4B), and the migration (Fig. 4C) of NP cells under periodic mechanical stress $(\mathrm{P}<0.05)$.

As shown in Fig. 4D, compared with the untransfected cells or the cells transfected with NC, transfection with integrin $\alpha 1$, siRNA led to a significant decrease in the phosphorylation of PLC $\gamma 1$ in the NPs under periodic mechanical stress $(\mathrm{P}<0.05)$.

\section{Discussion}

In NP cells, the ECM comprises predominantly Col-2 and proteoglycan, and the content gradually decreases with IDD (26). Cell migration is important for tissue reconstruction and repair (27). Anabolic ECM, predominantly Col2A1 and aggrecan, and the migration of NP cells are required for the elasticity and functions of IVD; therefore, the present study combined assessment of the expression levels of Col2A1 and proteoglycan with cell migration to investigate the regulatory effect of periodic mechanical stress on the functions of NP cells. The present study aimed to investigate the mechanisms underlying how periodic mechanical stress regulates the biological effects of NP cells.

Depending on the magnitude, frequency and duration of stress, NP cells exhibit diverse biological responses. Matsumoto et al (28) found that mechanical periodic stretch stress increased collagenous protein synthesis in rabbit NP cells. Neidlinger-Wilke et al (29) reported that low hydrostatic pressure $(0.25 \mathrm{MPa} ; 0.1 \mathrm{~Hz} ; 30 \mathrm{~min})$ promoted and high pressure (2.5 $\mathrm{MPa} ; 0.1 \mathrm{~Hz} ; 30 \mathrm{~min}$ ) decreased the expression levels of Col-2 and aggrecan in NP cells (29). Similarly, Hee et al (30) showed that the collagen and glycosaminoglycan contents were significantly higher in inner NP cells cultured under 0.2 $\mathrm{MPa}$ of compressive stress, compared with untreated control cells, but were significantly lower under $0.4 \mathrm{MPa}$ of compressive stress. In our previous study, it was found that periodic mechanical stress of $0-0.2 \mathrm{MPa}$ and $0.1 \mathrm{~Hz}$ not only significantly promoted the migration of chondrocytes (21), but also promoted the expression of ECM in the NP cells (23). Therefore, this condition was selected for investigation in the present study. The results demonstrated that soft periodic mechanical stress (0-0.2 Mpa; $0.1 \mathrm{~Hz}$; $6 \mathrm{~h})$ significantly induced the synthesis of ECM and the migration of NP cells, which were consistent with previous results $(23,28-30)$. Therefore, the results of the present and 
A
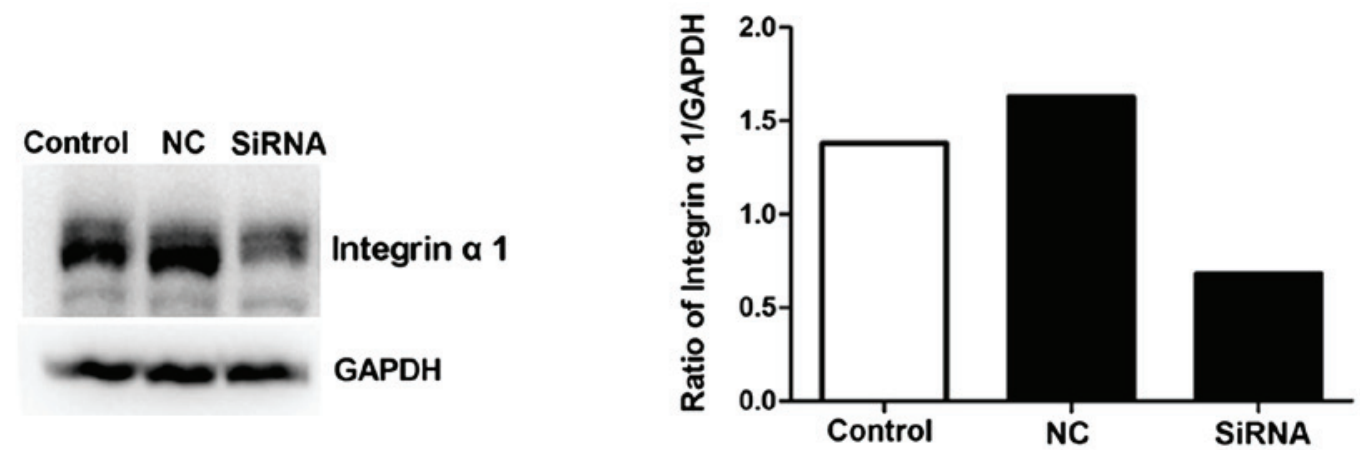

B

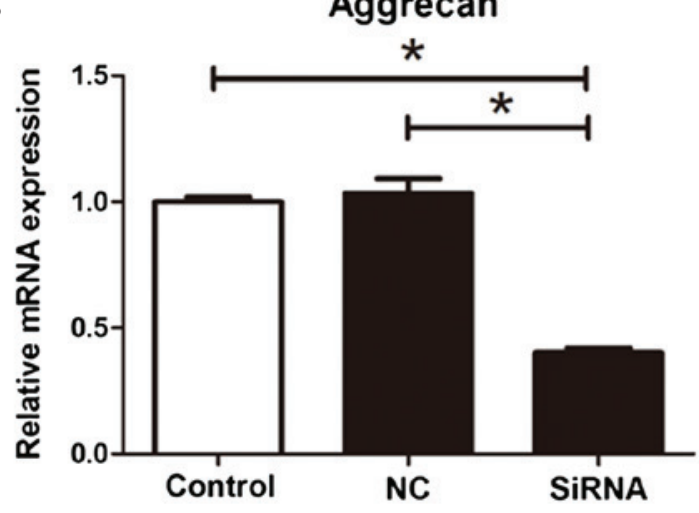

C

4h
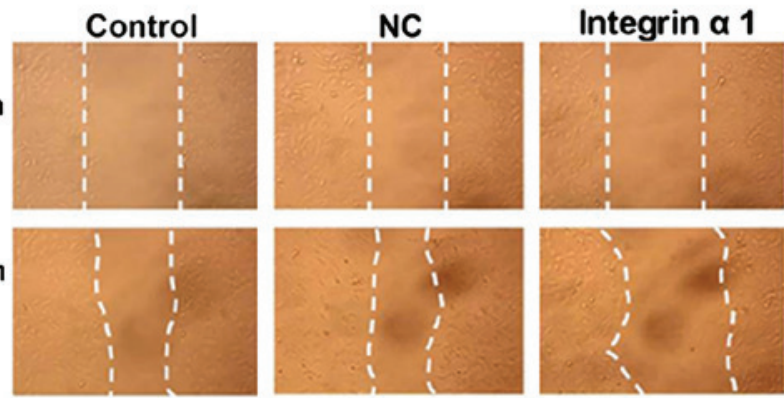
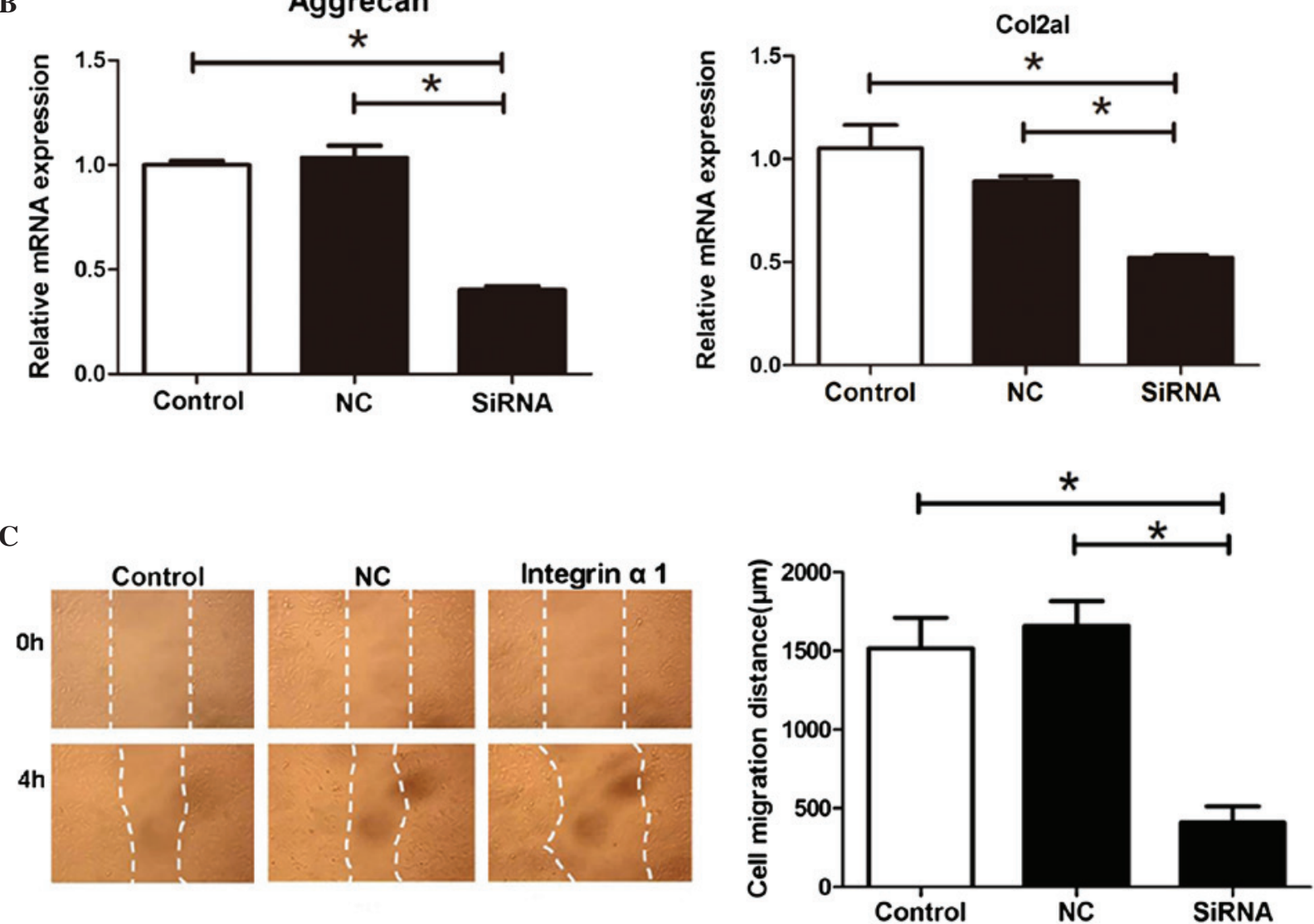

D
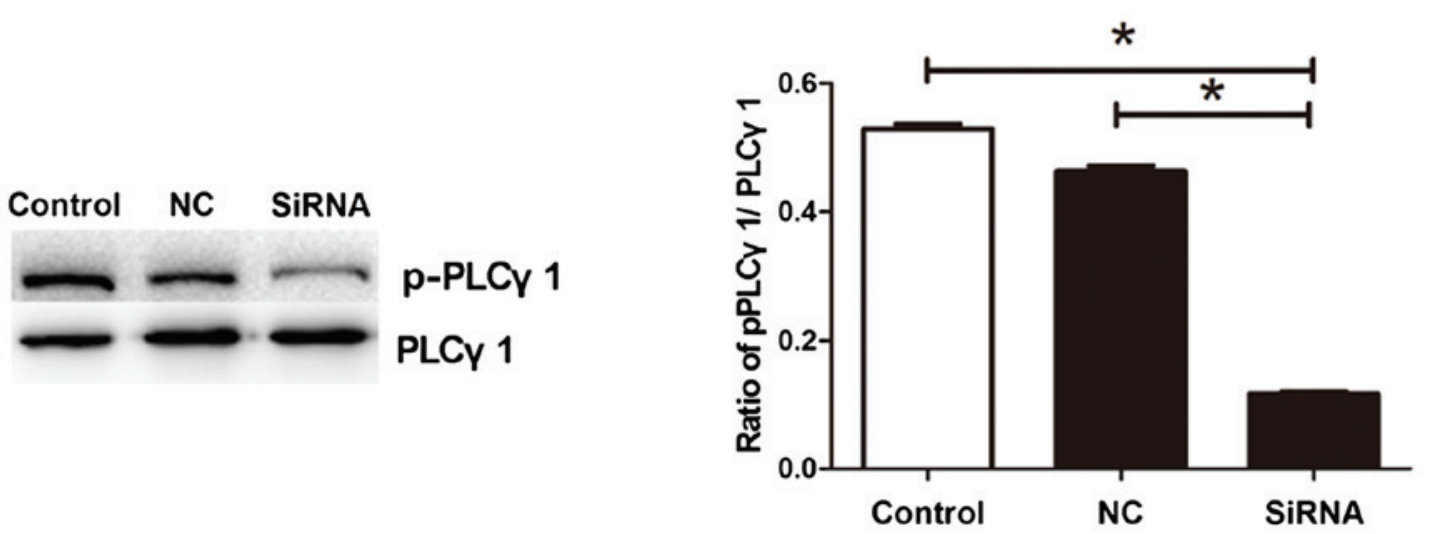

Figure 4. siRNA-based inhibition of the expression of integrin $\alpha 1$ suppresses the mRNA expression levels of Col2A1 and aggrecan, migration of NPs and phosphorylation of PLC- $\gamma 1$ under periodic mechanical stress. NP cells transfected with integrin $\alpha 1$ siRNA (siRNA), NC siRNA or untransfected cells (control) were collected. (A) Protein expression of integrin $\alpha 1$ was analyzed using western blot analysis. The NP cells from the siRNA, NC and control groups were collected to measure the (B) mRNA expression levels of Col2A1 and aggrecan, (C) migration (dotted lines indicate scratch widths; magnification, x200) and (D) phosphorylation of PLC- $\gamma 1$ under periodic mechanical stress. Data are expressed as the mean \pm standard deviation. "P $<0.05$, compared with the control or NC group ( $\mathrm{n}=3$ ). siRNA, small interfering RNA; Col2A1, collagen 2A1; PLC $\gamma 1$, phospholipase C- $\gamma 1$; p-PLC $\gamma 1$, phosphorylated PLC $\gamma 1$; NP, nucleus pulposus; $\mathrm{NC}$, negative control. 
previous studies indicated that soft stress can improve the biological functions of NPs.

Integrins mediate interactions with the ECM of NPs, which can promote cell attachment, survival and the biosynthesis of NPs. Inhibiting the $\beta 1$ subunit inhibits NP cell attachment to all substrates, whereas inhibiting subunits $\alpha 1, \alpha 2, \alpha 3$ and $\alpha 5$ simultaneously inhibit NP cell attachment to laminins (14). Fibronectin fragments or dynamic load $(1.3 \mathrm{MPa} ; 1.0 \mathrm{~Hz})$ induce the degeneration of NP cells and expression of integrin $\alpha_{5} \beta_{1}$, which is reversed by silencing the expression of integrin $\alpha_{5} \beta_{1}$ or by inhibiting its activity using the Arg-Gly-Asp peptide, indicating that integrin $\alpha_{5} \beta_{1}$ mediates the fibronectin fragment- or severe dynamic load-induced degeneration of NP cells and catabolic gene expression (17,31). However, until now, the effects of integrin $\alpha 1$ on the behaviors of NP cells under periodic mechanical stress, and the associated mechanisms, have not been previously reported.

The present study examined the variation in the expression of $\alpha 1, \alpha 5$ and $\alpha v$, the most important $\alpha$ subunits in NP cells (13), following periodic mechanical stress treatment. It was found that soft periodic mechanical stress increased the expression of integrin $\alpha 1$, decreased the expression of integrins $\alpha 5$ and $\alpha v$, induced the expression of ECM Col2A1 and aggrecan, and promoted the migration of NP cells. The siRNA-based inhibition of the expression of integrin $\alpha 1$ or treatment with the PLC $\gamma 1$ inhibitor, U73122, suppressed the ECM expression and the cell migration of the NPs under periodic mechanical stress, indicating that integrin $\alpha 1$ and PLC $\gamma 1$ are necessary for the effect of periodic mechanical stress on the NP cells. In addition, transfection of the NP cells with integrin $\alpha 1$ decreased the phosphorylation of PLC $\gamma 1$ at $\operatorname{Tyr}^{783}$, indicating that PLC $\gamma 1$ functions downstream of integrin $\alpha 1$. These results suggested that the integrin $\alpha 1$-mediated phosphorylation of PLC- $\gamma 1$ protein is involved in inducing the expression of ECM and migration of NP cells under soft cyclic stress. In addition, the decrease in the expression of $\alpha 5$ and $\alpha \mathrm{v}$ in NP cells in response to the periodic mechanical stress suggested that the $\alpha 5$ and $\alpha \mathrm{v}$ subunits function negatively in the behavior of the NP cells under periodic mechanical stress, consistent with previous reports $(17,31)$.

Various studies have shown that integrins regulate the functions of PLC $\gamma 1$. The activation of PLC $\gamma 1$ occurs following $\alpha 5 \beta 1$ integrin activation in fibroblasts, which is required for integrin-dependent adhesion (18). The inhibition of PLC $\gamma$ reduces $\alpha_{1} \beta_{1}$ integrin-mediated cell adhesion, indicating that PLC $\gamma$ is required for $\alpha_{1} \beta_{1}$-dependent signaling (32). It has also been shown that PLC $\gamma 1$ modulated $\beta 1$ integrin-dependent $\mathrm{T}$ lymphocyte migration on fibronectin (19). However, the interaction between integrin $\alpha_{1}$ and PLC $\gamma 1$ in NP cells has not been reported. On the basis of our previous investigation, which revealed that periodic mechanical stress induces the phosphorylation of PLC $\gamma 1$ in NP cells and the expression of ECM (23), the present study showed that PLC $\gamma 1$ phosphorylation (at $\mathrm{Tyr}^{783}$ ) was essential for the periodic mechanical stress-induced expression of ECM and the migration of NP cells, functioning downstream of integrin $\alpha 1$. How they interact to induce the expression of ECM and migration of NP cells under periodic mechanical stress requires further investigation.
Calcium is an important secondary messenger for cellular signal transduction in various physiological processes. It has been reported that stress induces the concentration of cellular calcium (33). Of note, PLC $\gamma 1$ is also involved in the regulation of cellular calcium concentrations $(34,35)$. Therefore, the regulation of cellular calcium concentrations may involve a mechanism by which PLC $\gamma 1$ mediates the periodic mechanical stress-induced expression of ECM and migration of NP cells. Further investigations are required to clarify this.

In conclusion, the present study demonstrated that periodic mechanical stress increased the expression of integrin $\alpha 1$ on the cell surface to induce the ECM expression and migration of NP cells, which was mediated by the expression of integrin $\alpha 1$ and the phosphorylation of downstream PLC $\gamma 1$. These results provide novel clues for further investigating the mechanisms underlying the effects of periodic mechanical stress on the behavior of NP cells, and understanding the pathogenesis and development of IDD.

\section{Acknowledgements}

This study was supported by the National Natural Science Foundation of China (grant no. 81201417).

\section{References}

1. Urban JP and Roberts S: Degeneration of the intervertebral disc. Arthritis Res Ther 5: 120-130, 2003.

2. Hwang PY, Chen J, Jing L, Hoffman BD and Setton LA: The role of extracellular matrix elasticity and composition in regulating the nucleus pulposus cell phenotype in the intervertebral disc: A narrative review. J Biomech Eng 136: 021010, 2014.

3. Wang C, Gonzales S, Levene H, Gu W and Huang CY: Energy metabolism of intervertebral disc under mechanical loading. J Orthop Res 31: 1733-1738, 2013.

4. Fernando HN, Czamanski J, Yuan TY, Gu W, Salahadin A and Huang CY: Mechanical loading affects the energy metabolism of intervertebral disc cells. J Orthop Res 29: 1634-1641, 2011.

5. Chan SC, Walser J, Käppeli P, Shamsollahi MJ, Ferguson SJ and Gantenbein-Ritter B: Region specific response of intervertebral disc cells to complex dynamic loading: An organ culture study using a dynamic torsion-compression bioreactor. PLoS One 8: e72489, 2013

6. Wilke HJ, Neef P, Caimi M, Hoogland T and Claes LE: New in vivo measurements of pressures in the intervertebral disc in daily life. Spine (Phila Pa 1976) 24: 755-762, 1999.

7. Kasra M, Goel V, Martin J, Wang ST, Choi W and Buckwalter J: Effect of dynamic hydrostatic pressure on rabbit intervertebral disc cells. J Orthop Res 21: 597-603, 2003.

8. Kuo YJ, Wu LC, Sun JS, Chen MH, Sun MG and Tsuang YH: Mechanical stress-induced apoptosis of nucleus pulposus cells: An in vitro and in vivo rat model. J Orthop Sci 19: 313-322, 2014.

9. Ernst N, Yay A, Bíró T, Tiede S, Humphries M, Paus R and Kloepper JE: $\beta 1$ integrin signaling maintains human epithelial progenitor cell survival in situ and controls proliferation, apoptosis and migration of their progeny. PLoS One 8: e84356, 2013.

10. Wang YC, Juan HC, Wong YH, Kuo WC, Lu YL, Lin SF, Lu CJ and Fann MJ: Protogenin prevents premature apoptosis of rostral cephalic neural crest cells by activating the $\alpha 5 \beta 1$-integrin. Cell Death Dis 4: e651, 2013.

11. Huang H, Kamm RD and Lee RT: Cell mechanics and mechanotransduction: Pathways, probes, and physiology. Am J Physiol Cell Physiol 287: C1-C11, 2004.

12. Hynes RO: Integrins: Bidirectional, allosteric signaling machines. Cell 110: 673-687, 2002.

13. Nettles DL, Richardson WJ and Setton LA: Integrin expression in cells of the intervertebral disc. J Anat 204: 515-520, 2004.

14. Bridgen DT, Gilchrist CL, Richardson WJ, Isaacs RE, Brown CR, Yang KL, Chen J and Setton LA: Integrin-mediated interactions with extracellular matrix proteins for nucleus pulposus cells of the human intervertebral disc. J Orthop Res 31: 1661-1667, 2013. 
15. Tran CM, Schoepflin ZR, Markova DZ, Kepler CK, Anderson DG, Shapiro IM and Risbud MV: CCN2 suppresses catabolic effects of interleukin-1 $\beta$ through $\alpha 5 \beta 1$ and $\alpha \mathrm{V} \beta 3$ integrins in nucleus pulposus cells: Implications in intervertebral disc degeneration. J Biol Chem 289: 7374-7387, 2014.

16. Yang SD, Ma L, Gu TX, Ding WY, Zhang F, Shen Y, Zhang YZ, Yang DL, Zhang D, Sun YP and Song YL: 17 $\beta$-Estradiol protects against apoptosis induced by levofloxacin in rat nucleus pulposus cells by upregulating integrin $\alpha 2 \beta 1$. Apoptosis 19: 789-800, 2014.

17. Kurakawa T, Kakutani K, Morita Y, Kato Y, Yurube T, Hirata H, Miyazaki S, Terashima Y, Maeno K, Takada T, et al: Functional impact of integrin $\alpha 5 \beta 1$ on the homeostasis of intervertebral discs: A study of mechanotransduction pathways using a novel dynamic loading organ culture system. Spine J 15: 417-426, 2015

18. Tvorogov D, Wang XJ,Zent R and Carpenter G: Integrin-dependent PLC-gammal phosphorylation mediates fibronectin-dependent adhesion. J Cell Sci 118: 601-610, 2005.

19. Shannon LA, Calloway PA, Welch TP and Vines CM: CCR7/CCL21 migration on fibronectin is mediated by phospholipase Cgamma1 and ERK1/2 in primary T lymphocytes. J Biol Chem 285: 38781-38787, 2010.

20. Zeng G, Cui X, Liu Z, Zhao H, Zheng X, Zhang B and Xia C: Disruption of phosphoinositide-specific phospholipases $C \gamma 1$ contributes to extracellular matrix synthesis of human osteoarthritis chondrocytes. Int J Mol Sci 15: 13236-13246, 2014

21. Nong L, Yin G, Ren K, Tang J and Fan W: Periodic mechanical stress enhances rat chondrocyte area expansion and migration through Src-PLCgamma1-ERK1/2 signaling. Eur J Cell Biol 89: 705-711, 2010

22. Ren K, Ma Y, Huang Y, Liang W, Liu F, Wang Q, Cui W, Liu Z, Yin G and Fan W: Periodic mechanical stress activates MEK1/2-ERK1/2 mitogenic signals in rat chondrocytes through Src and PLC $\gamma 1$. Braz J Med Biol Res 44: 1231-1242, 2011.

23. Xie H, Nong L, Gao G, Zhou D, He J and Chen X: Effect of the phospholipase $C-\gamma 1$ protein and its inhibitor on rat nucleus pulposus cells proliferation under periodic mechanical stress. Chin J Exp Sur 30: 2361-2363, 2013.

24. Risbud MV, Guttapalli A, Stokes DG, Hawkins D, Danielson KG, Schaer TP, Albert TJ and Shapiro IM: Nucleus pulposus cells express HIF-1 alpha under normoxic culture conditions: A metabolic adaptation to the intervertebral disc microenvironment. J Cell Biochem 98: 152-159, 2006.
25. Bron JL, Mulder HW, Vonk LA, Doulabi BZ, Oudhoff MJ and Smit TH: Migration of intervertebral disc cells into dense collagen scaffolds intended for functional replacement. J Mater Sci Mater Med 23: 813-821, 2012.

26. Roughley PJ: Biology of intervertebral disc aging and degeneration: Involvement of the extracellular matrix. Spine (Phila Pa 1976) 29: 2691-2699, 2004

27. Barreto Henriksson H, Lindahl A, Skioldebrand E, Junevik K, Tängemo $\mathrm{C}$, Mattsson $\mathrm{J}$ and Brisby $\mathrm{H}$ : Similar cellular migration patterns from niches in intervertebral disc and in knee-joint regions detected by in situ labeling: An experimental study in the New Zealand white rabbit. Stem Cell Res Ther 4: 104, 2013

28. Matsumoto T, Kawakami M, Kuribayashi K, Takenaka T and Tamaki T: Cyclic mechanical stretch stress increases the growth rate and collagen synthesis of nucleus pulposus cells in vitro. Spine (Phila Pa 1976) 24: 315-319, 1999.

29. Neidlinger-Wilke C, Würtz K, Urban JP, Börm W, Arand M, Ignatius A, Wilke HJ and Claes LE: Regulation of gene expression in intervertebral disc cells by low and high hydrostatic pressure. Eur Spine J 15 (Suppl 3): S372-S378, 2006.

30. Hee HT, Zhang J and Wong HK: An in vitro study of dynamic cyclic compressive stress on human inner annulus fibrosus and nucleus pulposus cells. Spine J 10: 795-801, 2010.

31. Xia $M$ and Zhu Y: Fibronectin fragment activation of ERK increasing integrin $\alpha_{5}$ and $\beta_{1}$ subunit expression to degenerate nucleus pulposus cells. J Orthop Res 29: 556-561, 2011.

32. Vossmeyer D, Hofmann W, Löster K, Reutter W and Danker K: Phospholipase Cgamma binds alphalbetal integrin and modulates alphalbeta1 integrin-specific adhesion. J Biol Chem 277: 4636-4643, 2002.

33. Browning JA, Saunders K, Urban JP and Wilkins RJ: The influence and interactions of hydrostatic and osmotic pressures on the intracellular milieu of chondrocytes. Biorheology 41: 299-308, 2004

34. Berridge MJ and Irvine RF: Inositol trisphosphate, a novel second messenger in cellular signal transduction. Nature 312: 315-321, 1984

35. Ji QS, Winnier GE, Niswender KD, Horstman D, Wisdom R, Magnuson MA and Carpenter G: Essential role of the tyrosine kinase substrate phospholipase C-gamma1 in mammalian growth and development. Proc Natl Acad Sci USA 94: 2999-3003, 1997. 